УДК 681.5

В.Н. Смолий

\title{
КОМПЛЕКСИРОВАНИЕ ТЕХНИЧЕСКИХ СРЕДСТВ ПРОИЗВОДСТВА ЭЛЕКТРОННЫХ АППАРАТОВ РАЗЛИЧНОГО НАЗНАЧЕНИЯ и УСЛОВИЙ эКСПЛУАТАЦИИ
}

Аннотация. Сформирован комплекс технических средств для технологического процесса производства блоков электронных аппаратов, позволяющий внедрить новые научно-исследовательские результаты в производство. Предложен критерий качества технологического процесса производства блока электронного аппарата, связывающий надежность выпускаемого изделия с его механическими, техническими и экономическими параметрами.

Ключевые слова: электронный аппарат, комплекс технических средств, технологический процесс производства, статистическая выборка, метод группового учета аргумента, автоматизированное управление, поддержка принятия решения, лицо принимающее решение.

\section{Введение}

Исследования, проводимые в отрасли производства электронных аппаратов (ЭА), показали наметившиеся тенденции глобализации взгляда на проблему повышения качества электронных средств, системности в решении проблемы повышения надежности за счет вариации техпроцессов изготовления электронных аппаратов и интеграции производства компьютерной техники по обеспечению связи моделирования с производством. В рассматриваемых системах моделирования электронных аппаратов недостаточное внимание уделяется вопросам анализа надежности электронных аппаратов с учетом влияния вибрационных, механических, компоновочных и прочих факторов. В связи с этим, актуальной является проблема развития теории автоматизации технологического процесса производства электронных аппаратов, связанная с наметившимися тенденциями в ее современном видении $[3,12]$.

Решить проблему повышения надежности ЭА невозможно без анализа влияния возмущающих факторов, таких, как вибрационные, механические и ударные воздействия на конструкторскую иерархию электронных аппаратов [1, 2]. Причем решение данной проблемы

\footnotetext{
${ }^{\circ}$ В.Н. Смолий
} 
предполагает выполнение последовательного анализа влияния этих возмущений с внесением корректив по результатам анализа. Целью внедрения вновь полученных результатов в производимый блок электронного аппарата служат, предложенные в работах [6, 7]: критерий компоновки типового элемента замены (ТЭЗ) ЭА, критерий компоновки блока ЭА и критерий качества технологического процесса (ТП) производства блока ЭА.

\section{Основное содержание и результаты работы}

Необходимо построить модель технологического процесса производства ЭА, которая будет связывать выходные параметры с вектором управления, в который будут входить надежностные, технические и экономические показатели всего процесса производства блока ЭА [4, 10]. Цель оптимизации - наилучшим образом обеспечить выполнение условий надежного функционирования блока ЭА.

Предложенные ранее критерии компоновки блока ЭА и ТЭЗ, как его составляющих, позволяют решить проблему обеспечения оптимальной устойчивости изделия к механическим, вибрационным и ударным воздействиям в детерминированной постановке задачи [5]. На данном этапе необходимо в стохастической постановке задачи обеспечить оптимальность протекания процесса производства с точки зрения повышения надежности ЭА, сокращения периода производства выпускаемого изделия, обеспечения экономического эффекта за счет исключения доводок и отбраковок выпускаемого изделия на последующих стадиях процесса производства из-за недоработок и скрытого брака на предыдущих стадиях процесса производства. Эти критерии позволяют посредством решения типизированных технологических задач (модифицированных разработанными ПТК для моделирования механических нагрузок ЭА и их составляющих) находить наилучшие последовательности выполнения технологических переходов и распределять найденные параметры и структуры на выпускаемое изделие.

Определение оптимального маршрута производства изделия с выделением участков и подразделений, ответственных за выполнение тех или иных параметров или структуры изделия будет рассмотрено в подразделе комплексирования технических средств для ТП производства блоков ЭА $[9,14]$. Оптимизация параметров производства изделия возможна посредством выделения и анализа таких составляющих критерия качества тех- 
нологического процесса производства блока ЭА, как: надежность выпускаемого изделия, время протекания процесса производства, влияние субъективного фактора на параметры и свойства изготавливаемого изделия (исключение субъективного фактора), экономическая целесо-образность внесения модификаций в существующий технологический процесс производства блока ЭА и его составляющих.

На увеличение критерия качества влияет надежность выпускаемого изделия, устойчивость изделия к механическим воздействиям, устойчивость изделия к вибрационным воздействиям, устойчивость изделия к ударным воздействиям [15]. Тенденции понижения величиины критерия качества ТП производства блока ЭА обусловлены: повышением стоимости изделия, увеличением затрат труда, пониже-ние скорости протекания ТП, необходимость дополнительных деталей (крепежа, прокладок и пр.), необходимость применения «уникальных» амортизаторов или «редких» схем их установки, необходимость применения нетипичных вариантов формовки выводов ЭРЭ.

В качестве основного критерия качества технологического процесса производства блока ЭА, из-за противоречивости влияния на него составляющих компонентов, выберем аддитивный критерий, имеющий следующий вид

$$
F(X)=\sum_{j=1}^{r} a_{j} y_{j}-\sum_{k=r+1}^{m} a_{k} y_{k},
$$

где $a_{j}$ и $a_{k}$ - весовые коэффициенты, первые $r$ параметров необходимо увеличивать, а оставшиеся $\mathrm{m}$ - r параметров - уменьшать.

Функцию (1) необходимо максимизировать, так как ищем наилучший вариант надежности выпускаемого изделия, при минимуме стоимости и времени протекания модифицированного технологического процесса.

Треть отказов всех электронных аппаратов происходят из-за механических воздействия, поэтому на основании статистических закономерностей представим надежность блока ЭА, как вероятность безотказной работы изделия и в виде комбинации устойчивости изделия к механическим, ударным и вибрационным воздействиям [13]. В этом случае надежность изделия будет величиной определяемой соотношением 


$$
P=e^{-\sum_{i=1}^{n} \lambda_{i} \cdot t}
$$

где $\lambda_{\mathrm{i}}$ - интенсивности отказов, обусловленные і фактором, к которым и отнесем отказы электронных аппаратов из-за механических, вибрационных и ударных воздействий.

Критерий качества технологического процесса производства ЭА представим в виде

$$
\mathrm{F}(\mathrm{x} 1, \mathrm{x} 2, \mathrm{x} 3, \mathrm{x} 4):=0.717 \mathrm{x} 1-0.1 \mathrm{x} 2-0.33 \times 3-0.2 \mathrm{x} 4 .
$$

Коэффициент при первом слагаемом (3) определяется (2), а остальные коэффициенты варьируются в зависимости от назначения изделия и объекта, на котором оно будет эксплуатироваться. Вид критерия качества и его предполагаемая величина для различных объектов приведены в табл. 1.

Ограничения, накладываемые на функционал качества, связаны со стоимостью обнаружения и устранения вредного влияния каждого из анализируемых факторов, выполняемых на ограниченном наборе управляющих воздействий на объект. Вид ограничений, накладываемых на функционал управления следующий:

$$
0.086 * \mathrm{x} 1+0.016 * \mathrm{x} 2+0.04 * \mathrm{x} 3+0.858 * \mathrm{x} 4 \leq 0.286 .
$$

Так как в ограничениях, накладываемых на функционал критерия качества, к примеру, невозможно суммировать время протекания процесса производства с экономической целесообразностью внесения изменений в существующий ТП производства ЭА, поэтому в (4) входят нормированные величины коэффициентов. Коэффициенты в (4) также «плавающие», что определяется спецификой объектов, на которых установлен ЭА и позволяет осуществлять подстройку ТП под условия эксплуатации изготавливаемого изделия.

В табл. 1 сведены предполагаемые веса каждого из составляющих критерия качества технологического процесса производства блока ЭА для изделий эксплуатируемых на различных объектах [11].

Предложенный критерий качества ТП производства блока ЭА позволил связать надежность выпускаемого изделия с его устойчивостью к механическим, вибрационным и ударным воздействиям, временем протекания процесса производства ЭА, с эффектом уменьшения влияния субъективного фактора, экономической целесообразностью внесения 
модификаций в существующий технологический процесс производства блока ЭА и его составляющих [16]. Получены группа ограничений, накладываемых на критерий качества, отражающих специфику объекта, на котором установлен ЭА. Получены сами значения критерия качества для различных объектов установки ЭА, что позволяет осуществлять функцию подстройки под условия эксплуатации и управлять ТП производства блока ЭА на основании четких правил.

таблица 1

Веса составляющих критерия качества ТП производства блока ЭА

\begin{tabular}{|l|c|c|c|c|c|}
\hline \multirow{2}{*}{ Тип объекта } & \multicolumn{4}{|c|}{$\begin{array}{c}\text { Составляющие критерия качества } \\
\text { технологического процесса }\end{array}$} & \multirow{2}{*}{$\begin{array}{c}\text { Величина } \\
\text { функционала } \\
\text { качества }\end{array}$} \\
\cline { 2 - 5 } & $\mathbf{1}$ & $\mathbf{2}$ & $\mathbf{3}$ & $\mathbf{4}$ & $\mathbf{2}$ \\
\hline Бытовой ЭА & $\mathbf{0 . 7 5}$ & $\mathbf{0 . 7}$ & $\mathbf{0 . 3 3}$ & $\mathbf{0 . 8}$ & 2.5 \\
\hline Стационарный ЭА & $\mathbf{0 . 9}$ & $\mathbf{0 . 1}$ & $\mathbf{0 . 3 3}$ & $\mathbf{0 . 2}$ & 3 \\
\hline Транспортный ЭА & $\mathbf{0 . 7 1 7}$ & $\mathbf{0 . 1}$ & $\mathbf{0 . 3 3}$ & $\mathbf{0 . 2}$ & 2.39 \\
\hline Военный ЭА & $\mathbf{0 . 6}$ & $\mathbf{0 . 7}$ & $\mathbf{0 . 9}$ & $\mathbf{0 . 8}$ & 2 \\
\hline Авиационный ЭА & $\mathbf{0 . 4 5}$ & $\mathbf{0 . 7}$ & $\mathbf{0 . 9}$ & $\mathbf{0 . 8}$ & 1.5 \\
\hline Космический ЭА & $\mathbf{0 . 1 5}$ & $\mathbf{0 . 7}$ & $\mathbf{0 . 9}$ & $\mathbf{0 . 8}$ & $\mathbf{0 . 5}$ \\
\hline
\end{tabular}

Для внедрения новых полученных научно-исследовательских результатов в существующий процесс производства блока электронного аппарата предлагается комплекс технических средств для ТП производства блоков ЭА. Для технологического процесса производства электронного аппарата характерно наличие нескольких блоков, выполняющих различные функции [4]. К таким блокам отнесем: анализ технического задания и генерация вариантов технического предложения, системотехнический, топологический, конструкторский, технологический, опытно-конструкторский, испытательный, производственный. Каждый из выделенных блоков работает со своим набором входных данных и генерирует соответствующие выходные данные. Все это происходит под влиянием управляющих воздействий с оптимизацией целевой функции. В рамках адаптивной системы по критерию компоновки, связывающему параметры ЭА и его составляющих с параметрами техпроцесса его производства.

В работе [5] было предложено комплексирование технических средств, базирующееся на организации процесса решения отдельной задачи в отдельной сети (структура отдела НИИ) с последующим объединением ее в общую сеть. Эта структура является громоздкой и 
экономически не эффективной в современных экономических условиях, поэтому применим структуру локальной сети с выходом в глобальную сеть, на которой реализуем функции организации процесса производства, включив сюда решение задачи экспертного рецензирования поступающих извне проектов и комплексную лабораторию механических испытаний. Предполагаемая структура комплекса технических средств приведена на рис. 1.

Разработанные программно-технические комплексы для моделирования механических нагрузок блока ЭА и его составляющих $[1,2]$ в предлагаемом комплексе технических средств выделены отдельным элементом. Посредством сетевой технологии реализована взаимосвязь разработанных программно-технических комплексов (ПТК), как с АРМами конструктора, технолога и производства, так и с экспериментальной установкой. Такой конфигурацией обеспечена возможность управления процессом производства и пополнения системы недостающей информацией, поступающей из лаборатории механических испытаний.

На рис. 1 компонент APM означает, либо одного специалиста, либо группу людей, выполняющих рассматриваемые функции. Автоматизированная складская система (АСC) связана, как с АРM технолога, так и с APM производства, что позволяет обеспечить «гибкость» процесса производства и уменьшить себестоимость изделия без потери надежности.

АРМ производства включен в единую локальную сеть и обуславливает течение технологического процесса, сходное с «естественным», однако в рассматриваемом случае на производственные станки и автоматы идут программы, перестроенные под задачу повышения надежности производимого изделия.

Отдельно выделенный блок - сеть Internet, предполагает выполнение предлагаемым комплексом технических средств функции рецензирования проектов, поступающих с других предприятий или фирм отрасли, и простоту организации и построения распределенной структуры производства, предполагающую и поддерживающую специализацию предприятий и расширяющие имеющееся в наличии у предприятий программно-техническое обеспечение. 


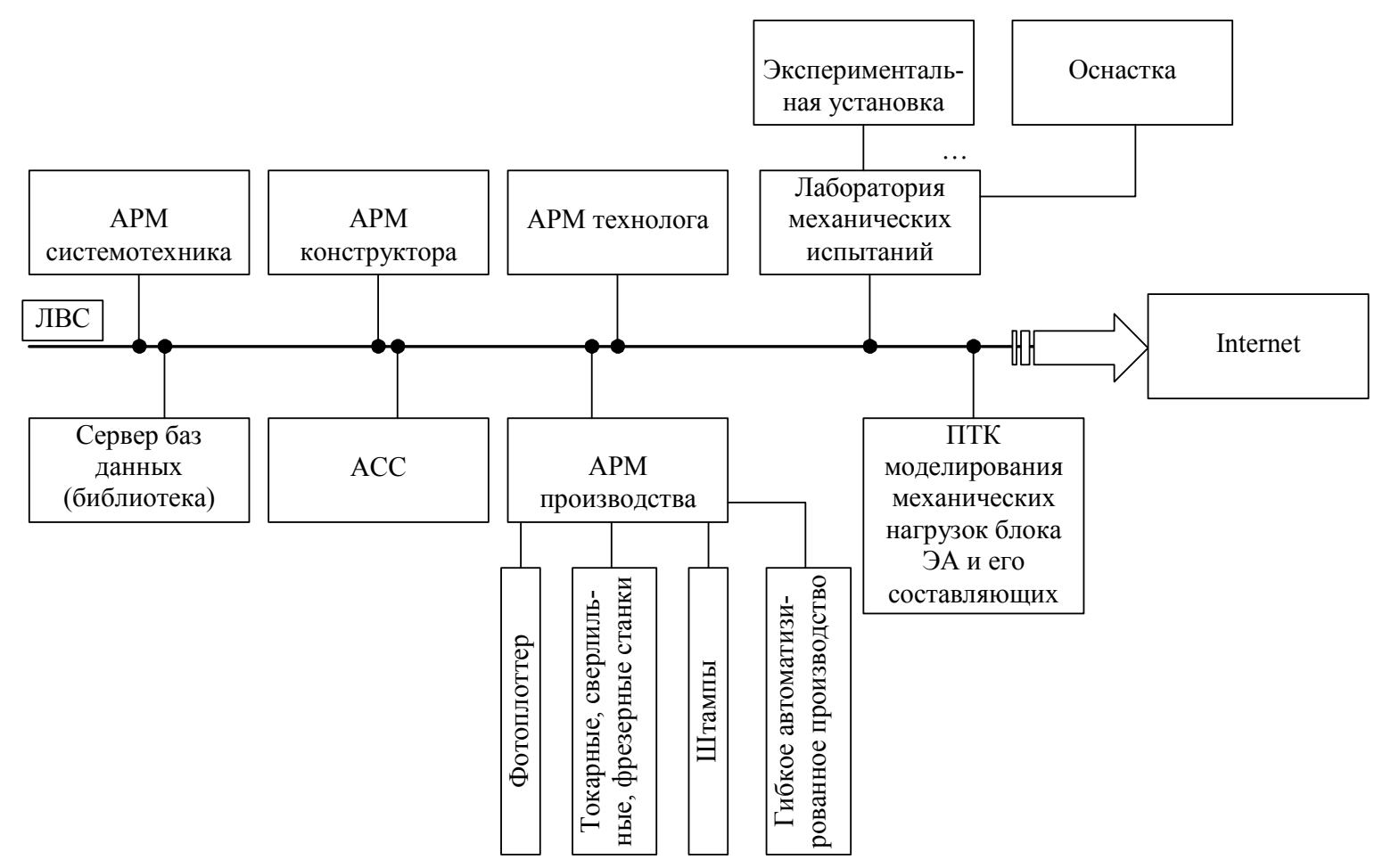

Рис. 1. Структура комплекса технических средств

Непосредственно решение задачи построения математической модели объекта управления - технологического процесса производства электронных аппаратов, включая формирование математического описания, проведение экспериментальных исследований по проверке адекватности и формирование программно-технических комплексов для моделирования механических нагрузок блоков электронных аппаратов и их составляющих приведено в [1]. Здесь проиллюстрирован также механизм идентификации свойства эмерджентности рассматриваемой системы на основании математического аппарата обнаружения и моделирования эффекта резонансного взаимодействия электрорадиоэлементов с печатной платой. Обобщение результатов моделирования и формирование на их основе подсистемы моделирования предлагаемой автоматизированной системы управления производством электронного аппарата проиллюстрировано в [2]. Работы [3] и [4] решают вопросы построения системы управления рассматриваемым технологическим процессом производства и внедрения полученных результатов в учебный процесс при изложении профессионально ориентированных дисциплин. В частности, для решения задачи оптимального управления рассматриваемым технологическим процессом, в работах предлагается внести модификацию в существующий процесс производства. 
Подобного рода внедрение влечет за собой необходимость обоснования эффективности предпринимаемых попыток на основании механизмов исследования выгод и издержек, что возможно путем применения метода анализа иерархий [5], [6]. Выгоды и издержки носят экономический и управленческий характер [7]. В частности примером экономических выгод выступает экономия средств за счет исключения отбраковок, экономических издержек - затраты на обучение персонала, управленческих выгод - формализация знаний и опыта экспертов, управленческих издержек - необходимость согласования выдаваемых системой рекомендаций с предполагаемым технологическим процессом производства. Содержание работ [5], [6] и [7] напрямую связано с решением задачи корреляции параметров технологического процесса в единый показатель его качества путем формирования функционала эффективности внедрения модификации в существующий технологический процесс производства на основании метода анализа иерархий.

С целью системного анализа поведения блоков электронных аппаратов следует разработанные математические описания их составляющих, как то: электрорадиоэлементов, печатных плат, типовых элементов замены и др. [1,2], интегрировать в математическое описание блоков ЭА и построить программно-технический комплекс, реализующий функции математического моделирования механических воздействий, в том числе резонансных, поведения блока электронного аппарата и его составляющих, их резонансного взаимодействия в процессе эксплуатации.

Разработанный программно-технический комплекс содержит два функциональных модуля: модуль "предварительного" исследования уравнения объекта и модуль моделирования механических нагрузок блоков ЭА с (без) системой виброизоляции. Первый модуль выполняет функции введения исходных данных и определения по ним корней уравнения объекта, а второй выполняет целый ряд процедур и функций, на описании которых остановимся более детально.

Процедуры и функции работы с матрицами реализуют программный код для выполнения операций формирования матриц, выделения диагональных элементов матриц, расчет определителей.

Функция преобразования чисел с плавающей запятой с двоичной точностью в строку содержит 84 строки программного кода и её назначением является преобразование используемых в программном 
модуле числовых данных в текстовую форму, необходимую для их вывода в виде "заголовков" элементов формы.

Процедуры описания основных компонентов формы и процедуры описания реакции на выбор элементов меню в формах, формы организуют интерфейс пользователя разрабатываемого ПТК.

Процедуры визуализации вспомогательных форм в модальном режиме обеспечивают создание, временное отображение и закрытие вспомогательных форм комплекса, а так же выполнение процедур, связанных с этими формами.

Процедуры реализации диалогового режима предназначены для задания (редактирования) в диалоговом режиме параметров исследуемого блока ЭА, условий его эксплуатации и параметров внешних воздействий.

Процедура задания реакции на нажатие кнопки «Очистить» инициализирует все используемые переменные начальными значениями и обнуляет данные, определяемые вычислениями комплекса.

Процедура реализации расчетных формул амплитудно-частотных характеристик, фазовых углов и собственных частот осуществляют вычисления математического описания исследуемого блока ЭА.

Процедура визуализации результатов осуществляет связь расчетных данных с элементами формы для их отображения.

Основные особенности использования ПТК. Для запуска ПТК моделирования механических нагрузок блоков электронных аппаратов необходимо выбрать из пакета VliVibBlokEA, файлы либо Project1.exе, либо vibro2.exе, после чего происходит запуск основной формы одного из модулей. На рис. 2 приведена основная форма первого модуля с рассчитанными корнями уравнения объекта. Форма содержит основное меню, состоящее из разделов: «Корни», «Собственные частоты», «Асимптоты», «Закрыть». Меню «Корни» позволяет определить корни уравнения объекта. Меню «Собственные частоты» определяет собственные частоты электронного аппарата. Меню «Асимптоты» определяет асимптоты для характеристик ЭА. Меню «Закрыть» завершает программу.

На рис. 3 изображен общий вид окна второго модуля разрабатываемого ПТК. Форма содержит основное меню, состоящее из разделов: «Файл», «Проект», «Сервис» и «Помощь», также в форме содержится область визуализации результатов вычислений амплитудно-частотных характеристик (АЧХ), два элемента для задания частотного диапазона 
при исследовании ЭА, указатели выбора проекции амплитуды на оси координат (в математическом описании ЭА рассматриваем как твердое тело с шестью степенями свободы), указатель для визуализации фазовых углов, непосредственно кнопка расчета АЧХ ЭА. Под графиком АЧХ предусмотрена кнопка вызова параметров воздействия, что позволяет анализировать условия эксплуатации исследуемого ЭА (в математическом описании учтено кинематическое и силовое возбуждение колебаний).

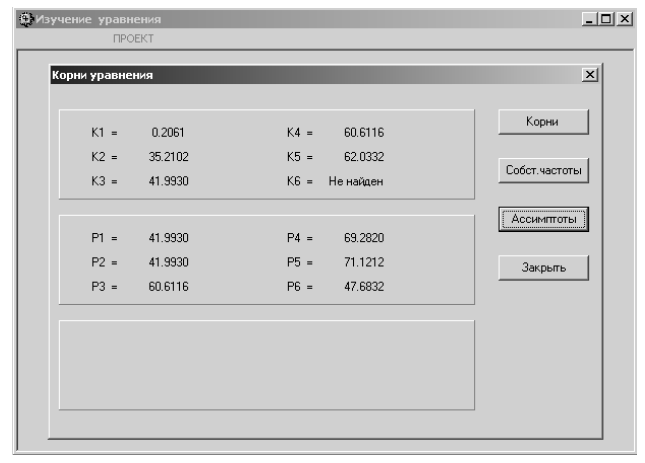

Рис. 2. Основная форма первого модуля

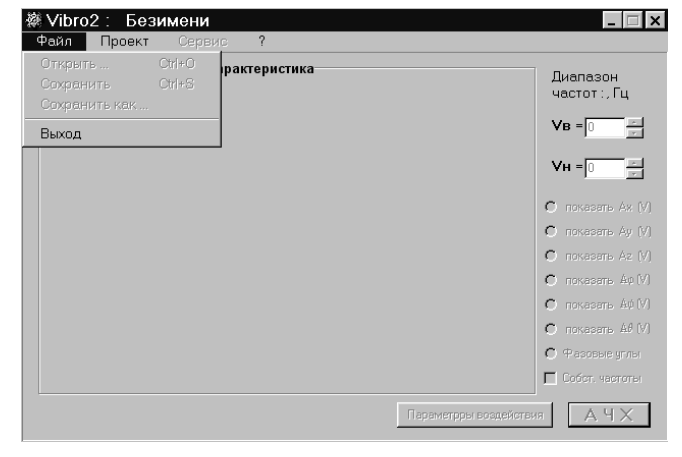

Рис. 3. Общий вид окна второго модуля

Меню «Файл» содержит следующие пункты: «Открыть», «Сохранить», «Сохранить как...»,«Выход». Первые пункты меню поз-воляют сохранить текущий проект или сохранить его под другим именем. По завершению работы с ПТК, пользователь, для выхода в операционную систему может воспользоваться стандартной кнопкой закрытия Windows - приложений или функцией «Выход» меню «Файл» .

Меню «Проект» (рис. 4) включает пункты: «Исходные данные», «Силовое воздействие» и «Кинематическое воздействие». При выборе пункта меню «Исходные данные» запускается форма (рис. 5), в которую необходимо внести исходные данные по исследуемому ЭА. К исходным данным отнесены, как параметры самого ЭА, так и параметры системы виброизоляции. Это сделано с целью обеспечения возможности моделирования механических характеристик ЭА, учета влияния условий эксплуатации на эти характеристики и последующего исправления вредного влияния внешних факторов посредством подбора системы виброизоляции в целом и параметров амортизаторов в отдельности. Такой подход позволяет решать задачу анализа объекта на этапе технического предложения процесса производства или проектирования блока ЭА, т.е. на ранних стадиях процесса синтеза 
нового изделия, что имеет высокую экономическую эффективность в современных условиях.

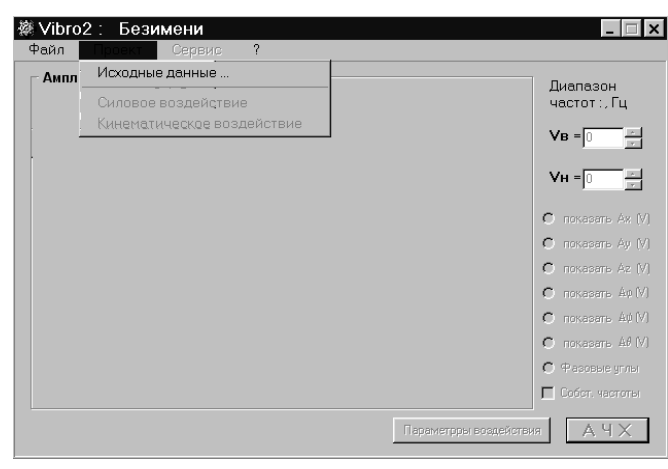

Рис. 4. Внешний вид меню «Проект» разрабатываемого ПТК

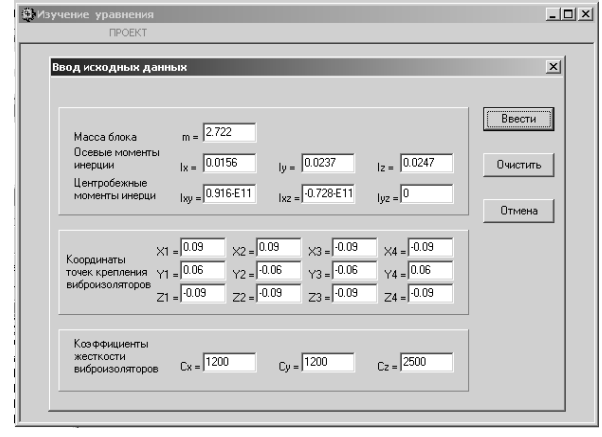

Рис. 5. Окно ввода исходных данных исследуемого объекта

Пункт основного меню «Сервис» содержит вспомогательную информацию по запуску программы предварительного исследования уравнения объекта и определения его характеристик, таких как: корни уравнения, собственные частоты, асимптоты и др.

Меню «Помощь» основной формы содержит справочную информацию по работе с пакетом.

К исходным параметрам блока ЭА отнесены также: координаты точек крепления виброизоляторов и коэффициенты жесткости виброизоляторов. Для учета разнообразия вариантов конструкций блоков ЭА и типов систем виброизоляции, варьирующихся в зависимости от назначения изделия, условий его эксплуатации и пр. объективных факторов, разрабатываемый ПТК предусматривает анализ следующих пяти схем виброизоляции: крепление блока ЭА к основанию и крышке (рис. 6), крепление блока ЭА к основанию (рис. 7), крепление блока ЭА к крышке (рис. 8), крепление блока ЭА к корпусу (рис. 9) и крепление блока ЭА к основанию и корпусу (рис. 10). Приведенные схемы взяты как типовые и могут быть реализованы на заданном числе виброизоляторов, на разных их типах и виброизоляторах с разными параметрами, что учтено в окне задания параметров системы виброизоляции (рис. 11).

Разрабатываемый ПТК для моделирования механических нагрузок блоков ЭА позволяет получить характеристики блока электронного аппарата (с отсутствующим влиянием условий эксплуатации) и его характеристики в определенных условиях эксплуатации, что дает возможность на ранних стадиях производства принять решение о необходимости применения системы виброизоляции и с помощью разработанного 
ПТК моделирования механических нагрузок блоков ЭА подобрать вид системы виброизоляции, типы и параметры виброизоляции.

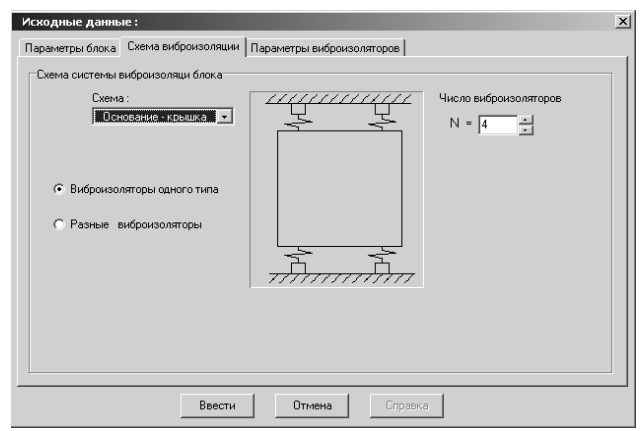

Рис. 6. Схема виброизоляции блока ЭА типа "основание-крышка"

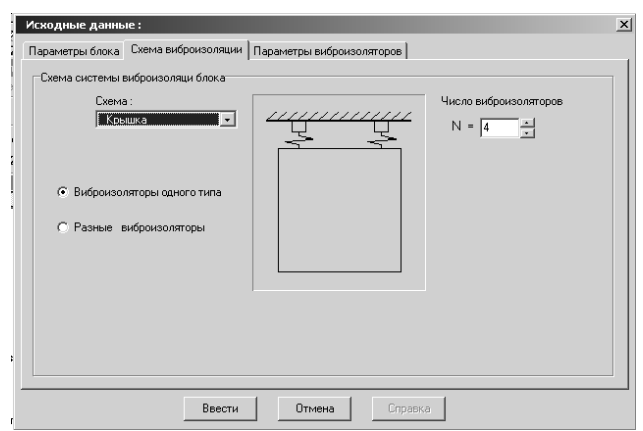

Рис. 8. Схема виброизоляции блока ЭА типа "крышка""

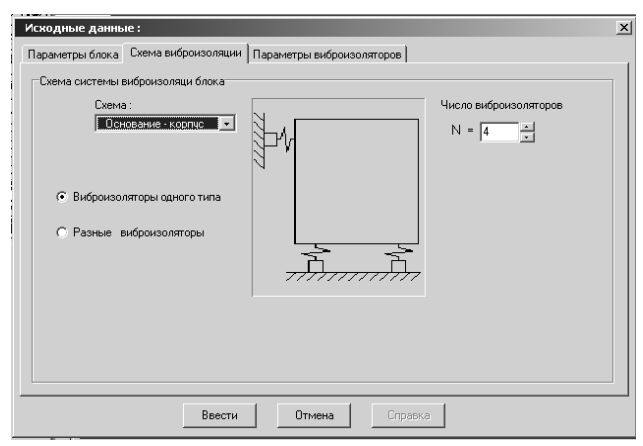

Рис. 10. Схема виброизоляции блока ЭА типа "основание-корпус"

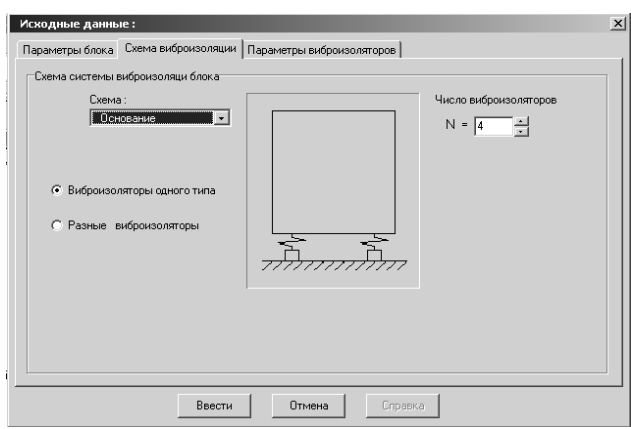

Рис. 7. Схема виброизоляции блока ЭА типа "основание"

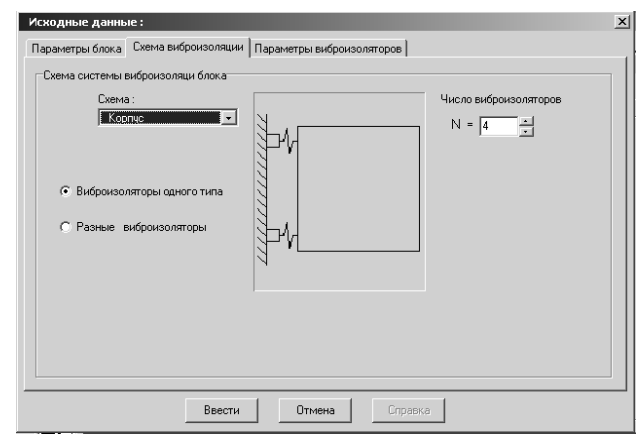

Рис. 9. Схема виброизоляции блока ЭА типа "корпус

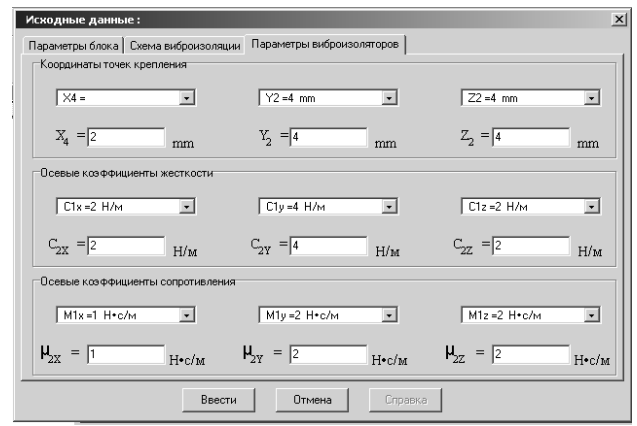

Рис. 11. Окно задания параметров системы виброизоляции

Окно визуализации результатов расчетов характеристик блока ЭА в виде таблицы результатов приведено на рис. 12 , а представление результатов расчетов в форме графиков приведено на рис. 13.

У разработанного ПТК имеется возможность (не в основном поле) отображать диапазон частот в котором производятся исследования ЭА, собственные частоты объекта и подсказывать тип отображаемой рассчитанной функции. 


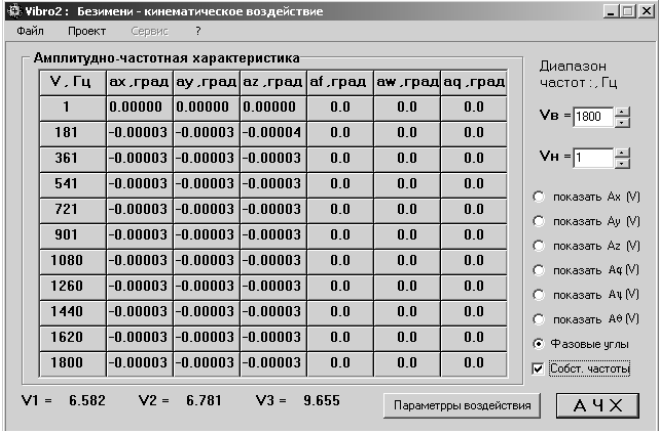

Рис. 12. Визуализации результатов расчетов в виде таблицы результатов

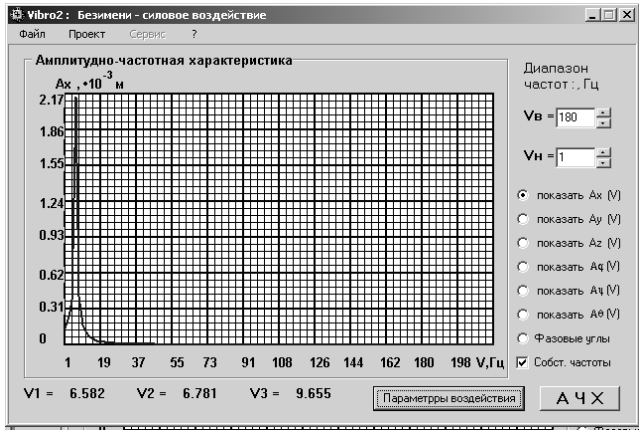

Рис. 13. Представление результатов расчетов в форме графиков

После получения результатов, имеется возможность сохранить их во внешнем файле и вывести на печать. Для реализации этих функций, пользователю необходимо, выйдя в основную оконную форму, вызвать меню «Файл» и выбрать необходимое подменю.

Для того, чтобы использовать разработанный ПТК в технологическом процессе производства электронной аппаратуры, необходимо проверить адекватность получаемых с помощью него результатов. Решение этой задачи связано с созданием экспериментальной установки, имитирующей блок ЭА и условия его работы. Внешний вид экспериментальной установки приведен на рис. 14. В состав экспериментальной установки входят: испытуемый блок ЭА; оснастка; вибростенд (ВЭДС-10 А), включающий блок измерения вибраций, блок генератора синусоидальных колебаний, блок усилителя, блок подмагничивания; измеритель шума и вибраций (ВШВ-003, 3 шт.); частотомер электронносчетный (Ч3-32); осциллограф электронносчетный (C1-79); осциллограф пишущий (НО71.1); фотобумага регистрирующая (УФ 87 100 200мм $\times 25 \mathrm{~m})$; строботахометр ТСт100; осветитель. Схема проведения экспериментальных исследований приведена на рис. 15.

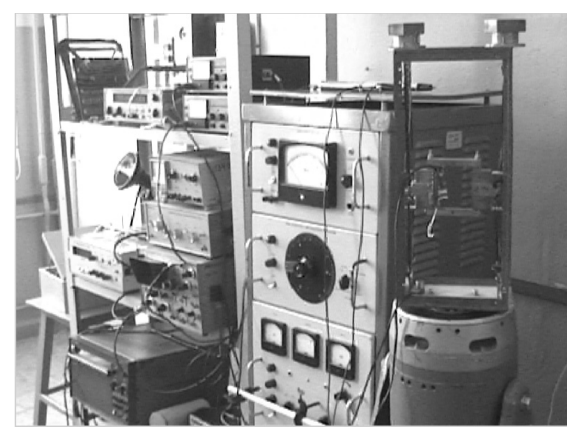

Рис. 14. Внешний вид экспериментальной установки

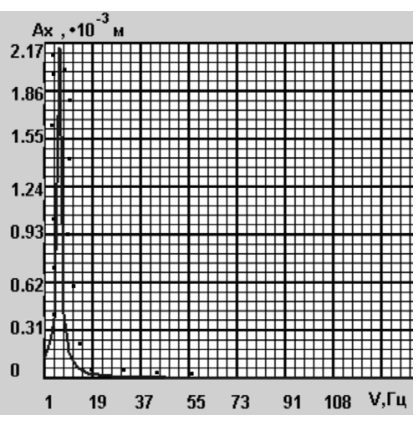

Рис. 15. Результаты моделирования и экспериментальных исследований 
Испытуемый блок ЭА в оснастке подвергается вибрационным воздействиям со стороны вибростенда. К испытуемому объекту подключена измерительная ветвь. Здесь посредством преобразования сигналов, снимаемых непосредственно с блока ЭА и с оснастки, возможна визуализация результатов на электронном осциллографе и фиксация последних на пишущем осциллографе. Сравнение этих сигналов позволяет точно вычислять механические характеристики блока ЭА и оснастки. Поддержка постоянным значения амплитуды колебаний оснастки позволяет повысить достоверность получаемых результатов. Осветитель строботахометра позволяет визуально наблюдать наличие резонансных процессов в испытуемом объекте и выяснить вид механических нагрузок, действующий на ЭА и их характер. Каждая серия испытаний, состоящая в последовательной записи процесса колебаний точек блока ЭА при поддержании постоянной амплитуды колебаний оснастки проводилась сначала при увеличении частоты, а затем при ее уменьшении. Каждая серия испытаний была повторена три раза.

На рис 14 приведен вид АЧХ блока ЭА, полученной с помощью разработанного ПТК и с помощью экспериментальных исследований. Произведя статистическую обработку результатов экспериментов, и сравнивая полученное значение дисперсии адекватности с дисперсией воспроизводимости по критерию Фишера, получаем что их отношение меньше табличного значения, что подтверждает адекватность разработанной математической модели блока ЭА.

\section{Выводы}

Предложенный критерий качества ТП производства блоков ЭА интегрирует механические, технические и экономические параметры производимого изделия, привязываясь к назначению аппаратуры и условиям эксплуатации, что дает возможность корректировать технологический процесс производства аппаратуры непосредственно по ходу выполнения операций. Рассмотренная структура комплекса технических средств отличается высокой гибкостью и эффективностью в современных экономических условиях, что достигается путем введения испытательных подразделений для сбора недостающей информации о параметрах объекта и разработанных ПТК моделирования, а также позволяет выполнять функции рецензирования проектов, поступающих с других предприятий или фирм отрасли. 
Разработаны программно-технические комплексы для моделирования механических нагрузок блоков электронных аппаратов и составляющих решают задачи моделирования электронного аппарата как объекта конструкторской подготовки производства и позволяют генерировать исходную информацию для разрабатываемой системы поддержки принятия решений и анализировать принимаемые решения конструкторской подготовки производства электронных аппаратов.

Разработан программно-технический комплекс для моделирования механических нагрузок электронных аппаратов, реализующий методику блиц-теста собственных частот конструкции блока электронного аппарата, моделирования механических нагрузок с (без) системой виброизоляции, расчета и визуализации параметров электронного аппарата. Комплекс выполняет функции моделирования и подготавливает информацию для реализации функции управления конструкторской подготовкой производства электронных аппаратов.

Предложена структурная схема управления конструкторской подготовкой производства электронных аппаратов, включающая сис-тему поддержки принятия решений, которая учитывает назначение и объект эксплуатации электронного аппарата, обеспечивает эффективность управления путем минимизации потерь времени и финансовых затрат на осуществление конструкторской подготовки производства электронного аппарата.

Разработанный критерий качества управления конструкторской подготовкой производства электронного аппарата (эффективность управления), взаимосвязанный с экспертными оценками и программно-техническими комплексами моделирования, запускает механизмы управления конструкторской подготовкой производства и обеспечивает качество, надежность, резонансную устойчивость и надежность электронного аппарата.

\section{ЛИТЕРАТУРА}

1. Смолий В.Н. Системное моделирование электронных аппаратов и компонентов // Адаптивні системи автоматичного управління. Міжвідомчий науково-технічний збірник. - К.:НТУУ «КПІ».,2015.№ 1(26).-С. 128 - 136.

2. Смолий В.Н. Особенности моделирования электронных аппаратов различного назначения и условий эксплуатации // Адаптивні системи автоматичного управління. Міжвідомчий науково-технічний збірник. - К.:НТУУ «КПІ».,2016.- № 1(28).-С. 116 - 128. 
3. Ульшин В.А., Смолий В.Н Программно-технический комплекс для моделирования механических нагрузок типовых элементов замены электронных аппаратов // Вісник СНУ ім. В.Даля. - 2005. - №10(92). C. 220-225.

4. Ульшин В.А., Смолий В.Н. Программно-технический комплекс для моделирования механических нагрузок блоков электронных аппаратов // Праці Луганського відділення Міжнародної Академії інформатизації. - 2006. - №1(12). - С. 45-49.

5. Смолий В.Н. Показатели эффективности управления процесса производства электронных аппаратов// Адаптивні системи автоматичного управління. Міжвідомчий науково-технічний збірник. К.:НТУУ «КПІ».,2012.- № 20(40).-С. 70 - 77.

6. Смолий В.Н. Нейросетевая технология обучения системы поддержки принятия решений для электронных аппаратов // Българско списание за инженерно проектиране, брой 23, юли 2014г. - С.125 - 135 .

7. В.Н. Смолий, А.В. Карпенко Концепция автоматизированной системы обучения для высшего учебного заведения// Проблеми інформаційних технологій. - Херсон.: ХНТУ., 2014. - № 15. - С.70-76.

8. Смолий В.Н. Управление производством сложноорганизованных технологических объектов//Вісник СНУ ім.В.Даля.- 2009. № 2 (132). Ч.2. - С. 46 - 55.

9. Смолий В.Н. Исследование эффективности управления процесса производства электронных аппаратов // Прогресивні технології i системи машинобудування: Міжнародний зб. наукових праць. Донецьк: ДонНТУ, 2010.- Вип. 39. - С. 174 - 178.

10. Смолий В.Н. Особенности концепции управления производством электронных аппаратов// Вісник СНУ ім.В.Даля. - 2010. № 2 (144). - C. 128 - 133.

11. Vitaly Ulshin, Victoria Smoliy. Automated management by designer preparation of production of electronic vehicles/ TEKA Kom. Mot. I Energ. Roln. - OL PAN, 2011, 11A, P.276 - 281.

12.12. Ульшин В.А., Смолий В.Н. Функции лица, принимающего решение, при управлении производством электронных аппаратов// Вісник СНУ ім.В.Даля. - 2011. - № 3 (157) - с. 214 - 220.

13. Смолій В.М. Синтез и исследование критерия компоновки типового элемента замены электронного аппарата/ Наукові праці Донецького національного технічного університету. Серія «Інформатика, 
кібернетика та обчислювальна техніка» (ІКОТ - 2012). - 2012.- Вип. 15 (203) - стор. 203-208.

14. Смолій В.М., Ульшин В.А., Фомін Я.Г. Mathematical design and operations of electronic vehicles management and introduction of results of researches in an educational pro cess. / TEKA. Commission of motorization and energetics in agriculture. -2012, Вип.12, №4, - стор. $285-289$.

15. Смолій В.М., Ульшин В.А. Принципиальная схема системы поддержки принятия решений конструкторской подготовки производства электронных аппаратов. / Науковий журнал. “Вісник” СНУім. В. Даля. - 2012, Вип. №3(174), ч.2. - с. 187-190.

16. Смолій В.М. Подсистемы СППР автоматизированного управления конструкторской подготовкой производства электронных аппаратовв машиностроении / Міжнародний збірник наукових праць. Прогресивні технології і системи машинобудування. -, 2012.- Вип. 43. - С. $272-277$. 